\title{
CONDITIONS FOR THE OSCILLATION OF SOLUTIONS OF ITERATIVE EQUATIONS
}

\author{
WIESŁAWA NOWAKOWSKA AND JAROSŁAW WERBOWSKI
}

Received 14 October 2002

We give some oscillation criteria for linear iterative functional equations. We compare obtained theorems with known results. We give applications to discrete equations too.

The problem of oscillation of solutions of differential and difference equations has been investigated by many authors since in the literature, there are many oscillation criteria for these equations (see $[2,5]$ ). However, for the iterative functional equations, the situation is different. Our aim is to give some new oscillation criteria for iterative functional equations. We are of the opinion that it is worth considering iterative functional equations because, in particular, they are recurrence equations which have a lot of applications. They can be used to describe processes in many areas such as biology, meteorology, economics, and so on (see [6]). This paper is concerned with the oscillatory solutions of linear iterative functional equations of the form

$$
Q_{0}(t) x(t)+Q_{1}(t) x(g(t))+Q_{2}(t) x\left(g^{2}(t)\right)+\cdots+Q_{m+1}(t) x\left(g^{m+1}(t)\right)=0, \quad m \geq 1,
$$

where $x$ is an unknown real-valued function and $Q_{k}: I \rightarrow \mathbb{R}$, for $k=0,1, \ldots, m+1$, and $g: I \rightarrow I$ are given functions, such that $\mathbb{R}$ is the set of real numbers and $I$ denotes an unbounded subset of $\mathbb{R}_{+}=[0, \infty)$. By $g^{m}$ we mean the $m$ th iterate of the function $g$, that is,

$$
g^{0}(t)=t, \quad g^{m+1}(t)=g\left(g^{m}(t)\right), \quad t \in I, m=0,1, \ldots
$$

By $g^{-1}$ we mean the inverse function of $g$ and $g^{-m-1}(t)=g^{-1}\left(g^{-m}(t)\right)$. In this paper, upper indices at the sign of a function will denote iterations. In each instance, we have the relation $g^{1}(t)=g(t)$. Exponents of a power of a function will be written after a bracket containing the whole expression of the function. We also assume that

$$
g(t) \neq t, \quad \lim _{t \rightarrow \infty} g(t)=\infty, \quad t \in I .
$$

Moreover, we assume that $g$ has an inverse function.

Copyright (C) 2004 Hindawi Publishing Corporation

Abstract and Applied Analysis 2004:7 (2004) 543-550

2000 Mathematics Subject Classification: 39B12

URL: http://dx.doi.org/10.1155/S1085337504306305 
By a solution of (1), we mean a function $x: I \rightarrow \mathbb{R}$ such that $\sup \left\{|x(s)|: s \in I_{t_{0}}=\right.$ $\left.\left[t_{0}, \infty\right) \cap I\right\}>0$ for any $t_{0} \in \mathbb{R}_{+}$and $x$ satisfies $I$ in $(1)$.

A solution $x$ of (1) is called oscillatory if there exists a sequence of points $\left\{t_{n}\right\}_{n=1}^{\infty}$, $t_{n} \in I$, such that $\lim _{n \rightarrow \infty} t_{n}=\infty$ and $x\left(t_{n}\right) x\left(t_{n+1}\right) \leq 0$ for $n=1,2, \ldots$ Otherwise it is called nonoscillatory.

The purpose of this paper is to obtain new oscillation criteria for (1). The analogous problem has been considered in $[1,7,9]$.

In this paper, we will use the following lemma.

Lemma 1 [9]. Consider the functional inequalities

$$
\begin{aligned}
& x\left(g^{s}(t)\right) \geq p(t) x\left(g^{s-1}(t)\right)+q(t) x\left(g^{m+1}(t)\right), \\
& x\left(g^{s}(t)\right) \leq p(t) x\left(g^{s-1}(t)\right)+q(t) x\left(g^{m+1}(t)\right),
\end{aligned}
$$

where $m \geq 1, s \in\{1, \ldots, m\}, p, q: I \rightarrow \mathbb{R}_{+}$, and $g$ satisfies condition (3). If

$$
\liminf _{I \ni t \rightarrow \infty} \sum_{i=0}^{m-s} q\left(g^{i}(t)\right) \prod_{j=1}^{m-s+1} p\left(g^{i+j}(t)\right)>\left(\frac{m-s+1}{m-s+2}\right)^{m-s+2},
$$

then the functional inequality (4) (resp., (5)) does not have positive (resp., negative) solutions for large $t \in I$.

It is easy to notice that the existence of oscillatory solutions of (1) is connected with the sign of the functions $Q_{i}(i=0,1, \ldots, m+1)$ on $I$. That either $Q_{i}(t)>0$ or $Q_{i}(t)<0$, for $i=0,1, \ldots, m+1$ and $t \in I$, implies that every solution of (1) oscillates. So, similarly as in our previous considerations (see, e.g., [9]), we will assume that in (1), one of the coefficients of $Q_{i}(i=1,2, \ldots, m)$ has the sign opposite to that of others, that is, there exists $s \in\{1, \ldots, m\}$ such that $Q_{s}(t)<0$ and $Q_{i}(t)>0, i \in\{0,1, \ldots, m+1\}-\{s\}$. So, we further assume that for some $s \in\{1,2, \ldots, m\}$,

$$
Q_{s}(t)<0, \quad Q_{i}(t) \geq 0, \quad i=0,1, \ldots, s-1, s+1, \ldots, m+1
$$

with

$$
Q_{s-1}(t), Q_{s+1}(t)>0 \quad \text { for } t \in I
$$

Without loss of generality, we may assume that $Q_{s}(t)=-1, t \in I$. Then (1) takes the form

$$
x\left(g^{s}(t)\right)=\sum_{k=0}^{s-1} Q_{k}(t) x\left(g^{k}(t)\right)+\sum_{k=s+1}^{m+1} Q_{k}(t) x\left(g^{k}(t)\right), \quad m \geq 1,
$$

where $s \in\{1,2, \ldots, m\}, Q_{i}(t) \geq 0(i=0,1, \ldots, s-1, s+1, \ldots, m+1)$, and $Q_{s-1}(t), Q_{s+1}(t)>$ 0 for $t \in I$.

As usual, we take $\sum_{j=k}^{r} a_{j}=0$ and $\prod_{j=k}^{r} a_{j}=1$, where $r<k$.

We start from the following theorem. 
THeOrem 2. Every solution of (9) is oscillatory if one of the following conditions hold:

$$
\liminf _{I \ni t \rightarrow \infty} A(g(t)) B(t)>\frac{1}{4}
$$

or

$$
\begin{aligned}
\limsup _{I \ni t \rightarrow \infty}\{ & A(g(t)) B(t)+A\left(g^{2}(t)\right) B(g(t)) \\
& \left.+A\left(g^{2}(t)\right) A\left(g^{3}(t)\right) B(g(t)) B\left(g^{2}(t)\right)\right\}>1,
\end{aligned}
$$

where

$$
\begin{aligned}
& A(t)=\sum_{k=0}^{s-1} Q_{k}(t) \prod_{j=2}^{s-k} Q_{s+1}\left(g^{-j}(t)\right), \\
& B(t)=\sum_{k=s+1}^{m+1} Q_{k}(t) \prod_{j=2}^{k-s} Q_{s-1}\left(g^{j}(t)\right) .
\end{aligned}
$$

Proof. Suppose that (9) has a nonoscillatory solution $x$ and let $x(t)>0$ for $t \in I_{t_{1}}, t_{1} \geq 0$. Then also, in view of assumption (3) about function $g, x\left(g^{i}(t)\right)>0, i \in\{1,2, \ldots, m+1\}$, and $t \in I_{t_{2}}, t_{2} \geq t_{1}$. Thus, from (9) we get

$$
x\left(g^{s}(t)\right) \geq Q_{i}(t) x\left(g^{i}(t)\right) \quad \text { for } i=0,1, \ldots, s-1, s+1, \ldots, m+1 .
$$

Hence, we have

$$
\begin{aligned}
x\left(g^{s}(t)\right) & \geq Q_{s+1}(t) x\left(g^{s+1}(t)\right), \\
x\left(g^{s-2}(t)\right) & \geq Q_{s+1}\left(g^{-2}(t)\right) x\left(g^{s-1}(t)\right) .
\end{aligned}
$$

From above we obtain

$$
x\left(g^{s-3}(t)\right) \geq Q_{s+1}\left(g^{-3}(t)\right) x\left(g^{s-2}(t)\right) \geq Q_{s+1}\left(g^{-3}(t)\right) Q_{s+1}\left(g^{-2}(t)\right) x\left(g^{s-1}(t)\right) .
$$

Thus,

$$
x\left(g^{k}(t)\right) \geq x\left(g^{s-1}(t)\right) \prod_{j=2}^{s-k} Q_{s+1}\left(g^{-j}(t)\right), \quad k=0,1,2, \ldots, s-2 .
$$

Similarly from inequality (13) we get

$$
\begin{gathered}
x\left(g^{s}(t)\right) \geq Q_{s-1}(t) x\left(g^{s-1}(t)\right), \\
x\left(g^{s+2}(t)\right) \geq Q_{s-1}\left(g^{2}(t)\right) x\left(g^{s+1}(t)\right) .
\end{gathered}
$$

Hence,

$$
\begin{gathered}
x\left(g^{s+3}(t)\right) \geq Q_{s-1}\left(g^{3}(t)\right) x\left(g^{s+2}(t)\right) \geq Q_{s-1}\left(g^{3}(t)\right) Q_{s-1}\left(g^{2}(t)\right) x\left(g^{s+1}(t)\right), \\
x\left(g^{k}(t)\right) \geq x\left(g^{s+1}(t)\right) \prod_{j=2}^{k-s} Q_{s-1}\left(g^{j}(t)\right), \quad k=s+2, \ldots, m+1 .
\end{gathered}
$$


Using now (16) and (19) in (9), we obtain

$$
x\left(g^{s}(t)\right) \geq A(t) x\left(g^{s-1}(t)\right)+B(t) x\left(g^{s+1}(t)\right),
$$

where $A$ and $B$ are given by (12). Thus, in view of condition (10) and Lemma 1, inequality (20) cannot possess positive solutions. We obtain a contradiction. Now we prove the second part of the theorem. From (20) for $i \in\{0,1,2\}$, we have

$$
\begin{gathered}
x\left(g^{s+i}(t)\right) \geq A\left(g^{i}(t)\right) x\left(g^{s+i-1}(t)\right)+B\left(g^{i}(t)\right) x\left(g^{s+i+1}(t)\right), \\
x\left(g^{s}(t)\right) \geq A(t) x\left(g^{s-1}(t)\right) .
\end{gathered}
$$

From above we obtain

$$
\begin{aligned}
& x\left(g^{s+2}(t)\right) \geq A\left(g^{2}(t)\right) x\left(g^{s+1}(t)\right), \\
& x\left(g^{s+3}(t)\right) \geq A\left(g^{3}(t)\right) x\left(g^{s+2}(t)\right) .
\end{aligned}
$$

Hence,

$$
x\left(g^{s+3}(t)\right) \geq A\left(g^{2}(t)\right) A\left(g^{3}(t)\right) x\left(g^{s+1}(t)\right) .
$$

Using the above inequality in (21) for $i=2$, we get

$$
x\left(g^{s+2}(t)\right) \geq A\left(g^{2}(t)\right) x\left(g^{s+1}(t)\right)+A\left(g^{2}(t)\right) A\left(g^{3}(t)\right) B\left(g^{2}(t)\right) x\left(g^{s+1}(t)\right) .
$$

Now applying inequalities (20) and (25) in (21) for $i=1$, we have

$$
\begin{aligned}
x\left(g^{s+1}(t)\right) \geq A(t) A(g(t)) x\left(g^{s-1}(t)\right) & \\
+ & \left\{A(g(t)) B(t)+A\left(g^{2}(t)\right) B(g(t))\right. \\
& \left.+A\left(g^{2}(t)\right) A\left(g^{3}(t)\right) B(g(t)) B\left(g^{2}(t)\right)\right\} x\left(g^{s+1}(t)\right), \\
x\left(g^{s+1}(t)\right) \geq\{ & A(g(t)) B(t)+A\left(g^{2}(t)\right) B(g(t)) \\
& \left.+A\left(g^{2}(t)\right) A\left(g^{3}(t)\right) B(g(t)) B\left(g^{2}(t)\right)\right\} x\left(g^{s+1}(t)\right) .
\end{aligned}
$$

Dividing both sides of the above inequality by $x\left(g^{s+1}(t)\right)$, we get a contradiction with (11). This completes the proof.

Remark 3. In the particular case when $I=\mathbb{N}$ and $g(n)=n+1$, from iterative functional equations, we obtain recurrence equations. So, results obtained in this paper can be applied to recurrence equations, too. For example, condition (10) applied to the secondorder linear difference equation of the form

$$
c(n) x(n+1)+c(n-1) x(n-1)=b(n) x(n)
$$


where $n \in \mathbb{N}, b, c: \mathbb{N} \rightarrow(0, \infty)$, gives the result obtained by Hooker and Patula in [4, Theorem 5]. However, condition (11) applied to (28) improves the result presented in [3, Theorem 2.3]. Namely, this theorem has the following form: if for some sequence $n_{k} \rightarrow \infty$,

$$
\frac{\left[c\left(n_{k}\right)\right]^{2}}{b\left(n_{k}\right) b\left(n_{k}+1\right)}+\frac{\left[c\left(n_{k}+1\right)\right]^{2}}{b\left(n_{k}+1\right) b\left(n_{k}+2\right)} \geq 1,
$$

then every solution of (28) is oscillatory. On the other hand, condition (11) applied to (28) has the form

$$
\limsup _{n \rightarrow \infty}\left\{\frac{[c(n)]^{2}}{b(n) b(n+1)}+\frac{[c(n+1)]^{2}}{b(n+1) b(n+2)}+\frac{[c(n+1)]^{2}}{b(n+1) b(n+2)} \frac{[c(n+2)]^{2}}{b(n+2) b(n+3)}\right\}>1 .
$$

If we consider (9) with $s=1, I=\mathbb{N}$, and $g(n)=n+1$, then from Theorem 2, we obtain conditions of $[8$, Theorems 5 and 6$]$.

Now we give another condition for the oscillation of all solutions of (9). It can be applied when Theorem 2 is not satisfied.

Theorem 4. Suppose that

$$
\begin{gathered}
A(g(t)) B(t) \geq \delta>0, \quad \delta<\frac{1}{4} \text { for } t \in I, \\
\limsup _{I \ni t \rightarrow \infty}\left\{A(g(t)) B(t)+A\left(g^{2}(t)\right) B(g(t))\right. \\
\left.+A\left(g^{2}(t)\right) A\left(g^{3}(t)\right) B(g(t)) B\left(g^{2}(t)\right)\right\}>1-\delta^{2},
\end{gathered}
$$

where $A$ and $B$ are as previously given. Then all solutions of (9) are oscillatory.

Proof. Let $x(t)>0$, for $t \in I_{t_{1}}, t_{1} \geq 0$, be a nonoscillatory solution of (9). Then, as in the proof of Theorem 2 for $t \in I_{t_{2}}, t_{2} \geq t_{1}$, inequalities (16) and (19) hold. So, inequality (20) is also true. Thus, for sufficiently large $t$, inequalities (21) and (26) are also satisfied. From (21) for $i=0$, we have

$$
\begin{aligned}
x\left(g^{s}(t)\right) & \geq B(t) x\left(g^{s+1}(t)\right), \\
A(g(t)) x\left(g^{s}(t)\right) & \geq A(g(t)) B(t) x\left(g^{s+1}(t)\right) .
\end{aligned}
$$

Using assumption (31) in the above inequality, we obtain

$$
A(g(t)) x\left(g^{s}(t)\right) \geq \delta x\left(g^{s+1}(t)\right) .
$$

The last inequality gives

$$
\begin{aligned}
A(t) x\left(g^{s-1}(t)\right) & \geq \delta x\left(g^{s}(t)\right), \\
A(t) A(g(t)) x\left(g^{s-1}(t)\right) & \geq \delta^{2} x\left(g^{s+1}(t)\right) .
\end{aligned}
$$


Now applying the last inequality in (26), we have

$$
\begin{aligned}
x\left(g^{s+1}(t)\right) \geq \delta^{2} x & \left(g^{s+1}(t)\right) \\
+ & \left\{A(g(t)) B(t)+A\left(g^{2}(t)\right) B(g(t))\right. \\
& \left.+A\left(g^{2}(t)\right) A\left(g^{3}(t)\right) B(g(t)) B\left(g^{2}(t)\right)\right\} x\left(g^{s+1}(t)\right) .
\end{aligned}
$$

Now dividing both sides of the above inequality by $x\left(g^{s+1}(t)\right)$, we obtain

$$
\begin{aligned}
1-\delta^{2} \geq\{ & A(g(t)) B(t)+A\left(g^{2}(t)\right) B(g(t)) \\
& \left.+A\left(g^{2}(t)\right) A\left(g^{3}(t)\right) B(g(t)) B\left(g^{2}(t)\right)\right\} .
\end{aligned}
$$

The last inequality contradicts assumption (32). Thus, the theorem is proved.

Remark 5. The theorems given in this paper are analogous to those presented in [9] but conditions given in both papers are independent. For example, from [9, Theorem 1], it follows that every solution of (9) is oscillatory if

$$
\liminf _{I \ni t \rightarrow \infty} \sum_{i=0}^{m-s} Q\left(g^{i}(t)\right) \prod_{j=1}^{m-s+1} P\left(g^{i+j}(t)\right)>\left(\frac{m-s+1}{m-s+2}\right)^{m-s+2}
$$

where

$$
\begin{aligned}
& P(t)=\sum_{k=0}^{s-2} Q_{k}(t) \prod_{l=2}^{s-k} Q_{s+1}\left(g^{-l}(t)\right)+Q_{s-1}(t), \\
& Q(t)=\sum_{k=s+1}^{m} Q_{k}(t) Q_{m+s-k+1}\left(g^{k-s}(t)\right)+Q_{m+1}(t) .
\end{aligned}
$$

In order to show the independence of conditions (10) and (38), we consider the following iterative functional equation:

$$
x(t+2)=\frac{1}{[t]^{2}} x(t)+\frac{4}{50 t} x(t+1)+\frac{15 t}{50} x(t+3)+[t]^{2} x(t+4), \quad t>0 .
$$

In this equation, $m=3, s=2$, and $g(t)=t+1$. Thus, condition (10) takes the form

$$
\begin{aligned}
& \liminf _{t \rightarrow \infty} {\left[Q_{0}(t+1) Q_{3}(t-1)+Q_{1}(t+1)\right]\left[Q_{3}(t)+Q_{4}(t) Q_{1}(t+2)\right] } \\
& \quad=\lim _{t \rightarrow \infty}\left[\frac{1}{[t+1]^{2}} \frac{15(t-1)}{50}+\frac{4}{50(t+1)}\right]\left[\frac{15 t}{50}+[t]^{2} \frac{4}{50(t+2)}\right]=\frac{361}{2500}<\frac{1}{4},
\end{aligned}
$$


and is not fulfilled. But the above-mentioned equation has only oscillatory solutions because for this equation, condition (38) has the form

$$
\liminf _{t \rightarrow \infty}\left[Q(t) P(g(t)) P\left(g^{2}(t)\right)+Q(g(t)) P\left(g^{2}(t)\right) P\left(g^{3}(t)\right)\right]>\left(\frac{2}{3}\right)^{3},
$$

where

$$
\begin{aligned}
& P(t)=Q_{1}(t)+Q_{0}(t) Q_{3}\left(g^{-2}(t)\right), \\
& Q(t)=Q_{3}(t) Q_{3}(g(t))+Q_{4}(t),
\end{aligned}
$$

and is satisfied because

$$
\begin{aligned}
\lim _{t \rightarrow \infty}\{ & {\left[\frac{15 t}{50} \frac{15(t+1)}{50}+[t]^{2}\right]\left[\frac{1}{[t+1]^{2}} \frac{15(t-1)}{50}+\frac{4}{50(t+1)}\right] } \\
& \times\left[\frac{1}{[t+2]^{2}} \frac{15 t}{50}+\frac{4}{50(t+2)}\right] \\
& +\left[\frac{15(t+1)}{50} \frac{15(t+2)}{50}+[t+1]^{2}\right]\left[\frac{1}{[t+2]^{2}} \frac{15 t}{50}+\frac{4}{50(t+2)}\right] \\
& \left.\times\left[\frac{1}{[t+3]^{2}} \frac{15(t+1)}{50}+\frac{4}{50(t+3)}\right]\right\} \\
= & 0.314792>\left(\frac{2}{3}\right)^{3} .
\end{aligned}
$$

Now we consider the iterative functional equation of the form

$$
x(t+2)=\frac{1}{5[t]^{2}} x(t)+\frac{1}{4 t} x(t+1)+\frac{3 t}{5} x(t+3)+\frac{3[t]^{2}}{5} x(t+4), \quad t>0 .
$$

The above-mentioned equation possesses only oscillatory solutions too. For this equation, condition (38) is not true but condition (10) is satisfied.

\section{References}

[1] W. Golda and J. Werbowski, Oscillation of linear functional equations of the second order, Funkcial. Ekvac. 37 (1994), no. 2, 221-227.

[2] I. Györi and G. Ladas, Oscillation Theory of Delay Differential Equations with Applications, Oxford Mathematical Monographs, The Clarendon Press, Oxford University Press, New York, 1991.

[3] J. W. Hooker, M. K. Kwong, and W. T. Patula, Oscillatory second order linear difference equations and Riccati equations, SIAM J. Math. Anal. 18 (1987), no. 1, 54-63.

[4] J. W. Hooker and W. T. Patula, Riccati type transformations for second-order linear difference equations, J. Math. Anal. Appl. 82 (1981), no. 2, 451-462.

[5] G. S. Ladde, V. Lakshmikantham, and B. G. Zhang, Oscillation Theory of Differential Equations with Deviating Arguments, Monographs and Textbooks in Pure and Applied Mathematics, vol. 110, Marcel Dekker, New York, 1987. 
[6] V. Lakshmikantham and D. Trigiante, Theory of Difference Equations. Numerical Methods and Applications, Mathematics in Science and Engineering, vol. 181, Academic Press, Massachusetts, 1988.

[7] W. Nowakowska and J. Werbowski, Oscillation of linear functional equations of higher order, Arch. Math. (Brno) 31 (1995), no. 4, 251-258.

[8]_, Oscillatory behavior of solutions of linear recurrence equations, J. Differ. Equations Appl. 1 (1995), no. 3, 239-247.

[9] Oscillatory behavior of solutions of functional equations, Nonlinear Anal. 44 (2001), no. $6,767-775$.

Wiesława Nowakowska: Institute of Mathematics, Poznań University of Technology, 60-965 Poznań, Poland

E-mail address: wnowakow@math.put.poznan.pl

Jarosław Werbowski: Institute of Mathematics, Poznań University of Technology, 60-965 Poznań, Poland

E-mail address: jwerbow@math.put.poznan.pl 


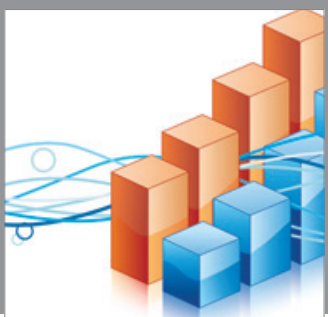

Advances in

Operations Research

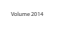

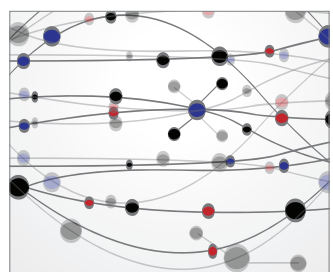

\section{The Scientific} World Journal
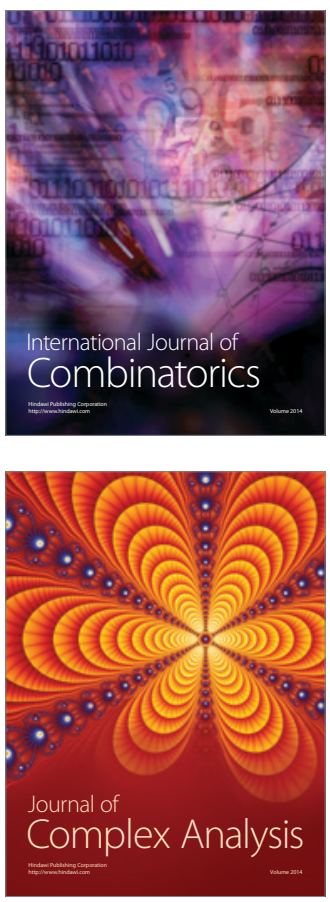

International Journal of

Mathematics and

Mathematical

Sciences
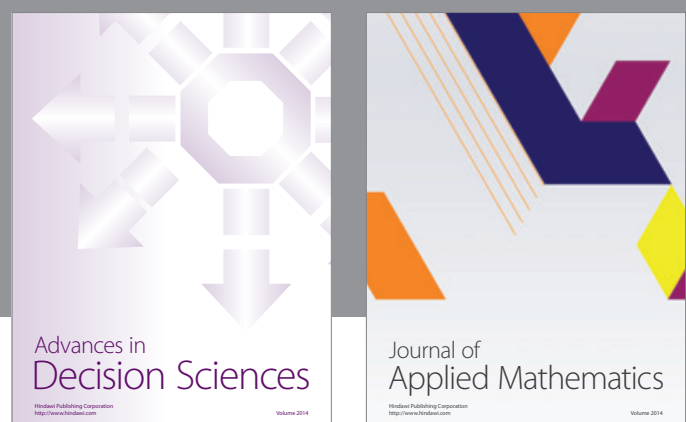

Journal of

Applied Mathematics
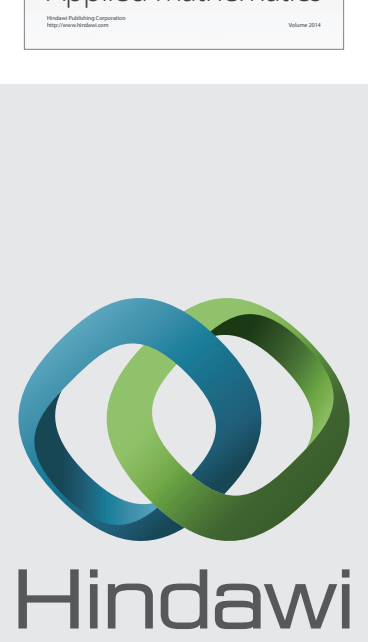

Submit your manuscripts at http://www.hindawi.com
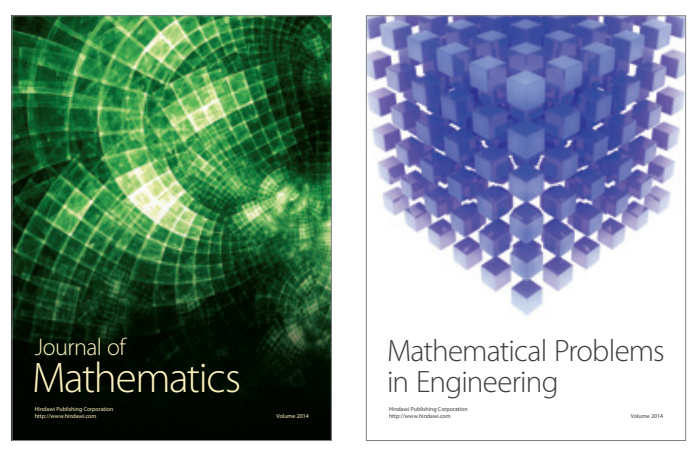

Mathematical Problems in Engineering
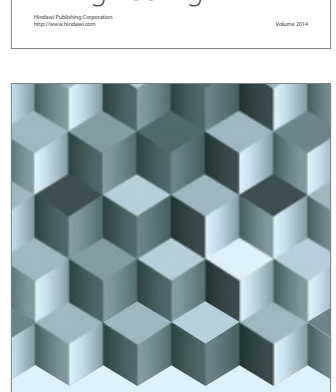

Journal of

Function Spaces
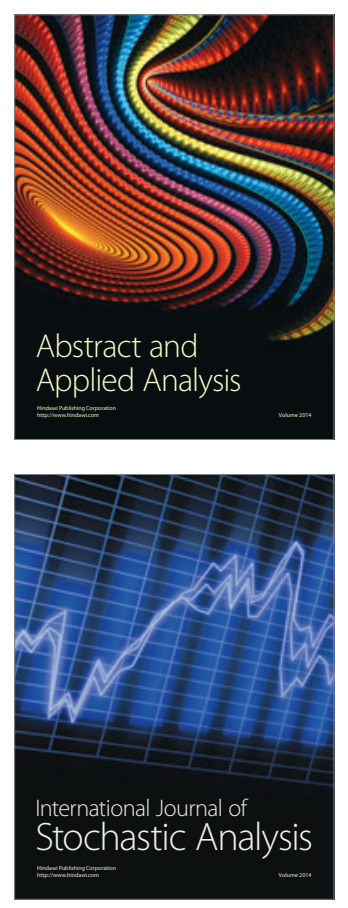

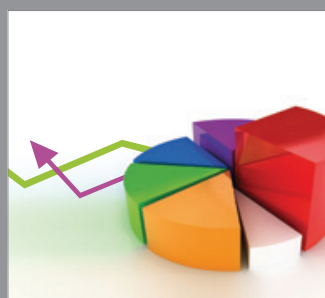

ournal of

Probability and Statistics

Promensencen
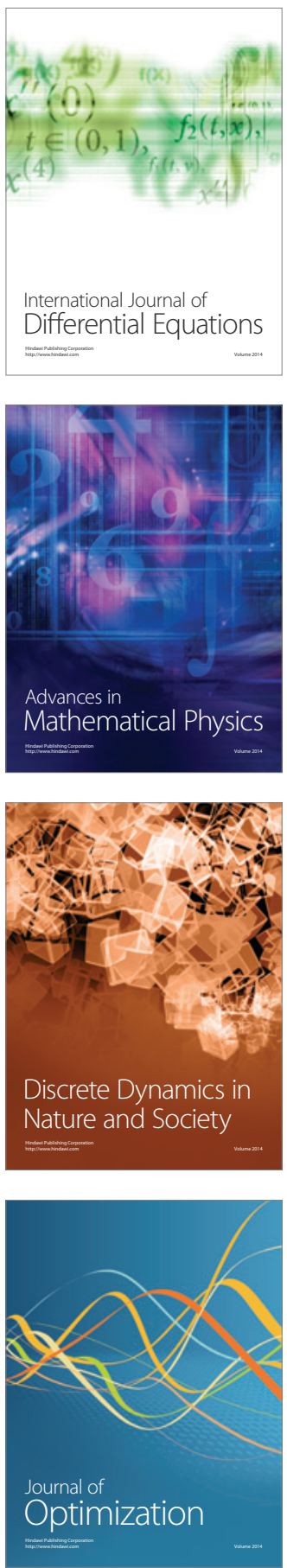